\title{
BUNCH-LENGTH MONITOR FOR AN ELECTRON LINAC
}

\author{
M.I. Ayzatsky, E.Z. Biller, A.N. Dovbnya, I.V. Khodak, V.A. Kushnir, V.V. Mitrochenko, \\ A.N. Opanasenko, S.A. Perezhogin, D.L. Stepin, L.M. Zavada \\ National Science Center 'Kharkov Institute of Physics \& Technology' \\ 1 Academicheskaya St., Kharkov, 61108, Ukraine, \\ V.K. Kiseliov, Ye.M. Kuleshov, M.S.Yanovsky \\ Usikov Institute for Radiophysics and Electronics NASU \\ 12 Ac. Proskura St, Kharkov, 61085, Ukraine
}

\begin{abstract}
We report the design of the bunch length monitor that is based on generation of coherent Smith - Purcell radiation (CSPR) by relativistic electron bunches that pass over a metallic grid. The monitor consists of grating and receiving equipment. The preliminary experimental data on CSPR in millimeter wave range are described. The experiments were carried out on the S-band linac LIC (Laser Injector Complex) with $13 \mathrm{MeV}$ electron beam. The millimeter wave radiation has been studied in perpendicular direction to the grating plane. It has been shown that CSPR can be easily used for the linac adjustment
\end{abstract}

\section{THEORETICAL CONSIDERATIONS}

It is known that an electron beam moving near discontinuities generates electromagnetic radiation. If discontinuities are placed periodically along a beam trajectory (for example a diffraction grating) the radiation has specific characteristics and it was named as SmithPurcell radiation in honor of scientists who observed such radiation for the first time [1]. Such radiation is a subject of investigation at many research centers (see for example [2]). It was determined that the radiation wavelength $\lambda$ depends on observing angle $\theta$ as following:

$$
\lambda(\theta)=\frac{d}{|n|}\left(\frac{1}{\beta}-\cos (\theta)\right),
$$

where $\beta$ is normalised electron velocity, $n$ is diffraction order and $d$ is grating period.

The spectral intensity depends exponentially on the parameter $b$ (distance between a beam axis and the highest point on the grating surface) $P(\lambda) \sim \exp (-$ $4 \pi b /(\lambda \gamma \beta)$ ) (where $\gamma$ is Lorenz factor) that is featured for the given radiation type [3]. Regardless of a grating profile the maximum of intensity of incoherent radiation of relativistic particles is in a short-wave part of a spectrum at observation angles close to $\theta \sim 1 / \gamma$. The radiation coherent component is observed in wavelength range larger than bunch sizes when a grating is excited by relativistic electron bunches with linear sizes less or compared with the period $d$. This causes the significant increasing of the long-wave radiation intensity and changing its angular distribution [4]. In general case the radiation intensity in a solid angle $d \Omega$ can be represented as following:

$$
\frac{d P}{d \Omega}=N \frac{d P_{0}(\theta)}{d \Omega} \exp \left(-\frac{4 \pi b}{\lambda(\theta) \beta \gamma}\right)[1+(N-1) F(\lambda(\theta))],
$$

where $d P_{0}(\theta) / d \Omega$ is the angular intensity distribution of the spontaneous radiation of a single electron moving with zero parameter $b$ over the grating; $F(\lambda)$ is the bunch form-factor (the spatial Fourier component in particle density average distribution in a bunch). For the bunch with Gauss distribution it follows $F(\lambda)=\exp \left[-\left(2 \pi \sigma_{z} / \lambda\right)^{2}\right]$. In this work we investigate the coherent radiation observed in the orthogonal direction to the grating surface $\left(\theta=90^{\circ}\right.$, so $\lambda=d$ as it follows from Eq. (1)). Fig. 1 illustrates dependence of the ratio of the intensity density of the radiation to the intensity density of the incoherent radiation on the grating period for various values of a bunch length $\sigma_{z}$. Calculations were carried out for the bunch with $N \cong 1.8 \quad 10^{9}$ electrons and energy of $13 \mathrm{MeV}$.

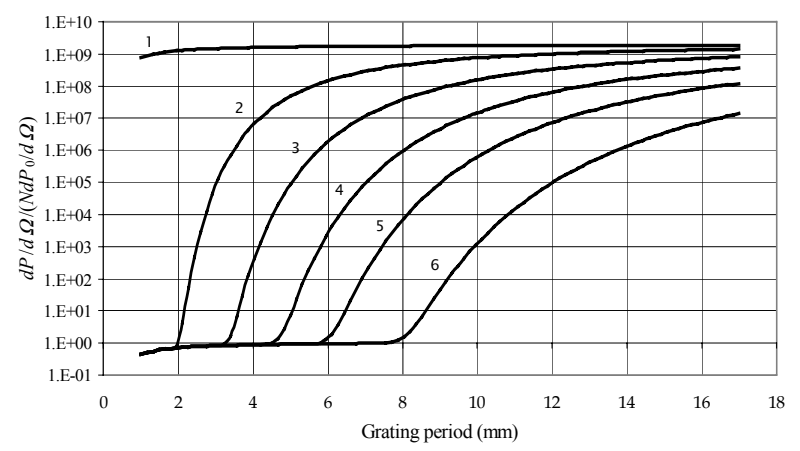

Figure 1: Relative density of the diffraction radiation intensity; $1-\sigma_{z}=0,2-\sigma_{z}=1.5 \mathrm{~mm}, 3-\sigma_{z}=2.5 \mathrm{~mm}, 4$ $\sigma_{z}=3.5 \mathrm{~mm}, 5-\sigma_{z}=4.5 \mathrm{~mm}, 6-\sigma_{z}=6 \mathrm{~mm}$.

The strong dependence of coherent radiation intensity on a bunch length can be used for the bunch length monitoring. Thus, for $\sigma_{z} / \lambda$ varying form 0.2 to 0.75 the radiation intensity is decreased by factor of $10^{9}$ down to incoherent radiation threshold. Estimations showed that for the bunch with $N \cong 1.8 \quad 10^{9}$ passing with zero parameter $b$ over the grating with $8 \mathrm{~mm}$ period the power density is $N d P_{0}(\theta) / d \Omega \approx 1.2 \mu W / s r$.

\section{MONITOR}

Proposed monitor consists of a copper diffracting grating equipped with the gear for the diffraction grating 
movement and the secondary emission monitor for the measurement of the beam transverse distribution. Radiation is extracted from a vacuum chamber through a sapphire window. The gear provides movement of the grating in perpendicular direction to the beam axis to change distance between the beam axis and the grating as well as the grating rotation to change an effective grating period. The radiation is received by a horn aerial placed at $1 \mathrm{~m}$ from the beam axis and detected by the calibrated detector. The H-plane of the aerial coincides with the flute plane of the diffraction grating. Pulse signals that are proportional to quantity of electrons falling on the secondary emission monitor and the grating as well as grating position and a pulse signal from the detector are measured by the computerised control system to be observed, stored and processed. The experiment set-up layout is illustrated in the Fig. 2 in a simplified way.

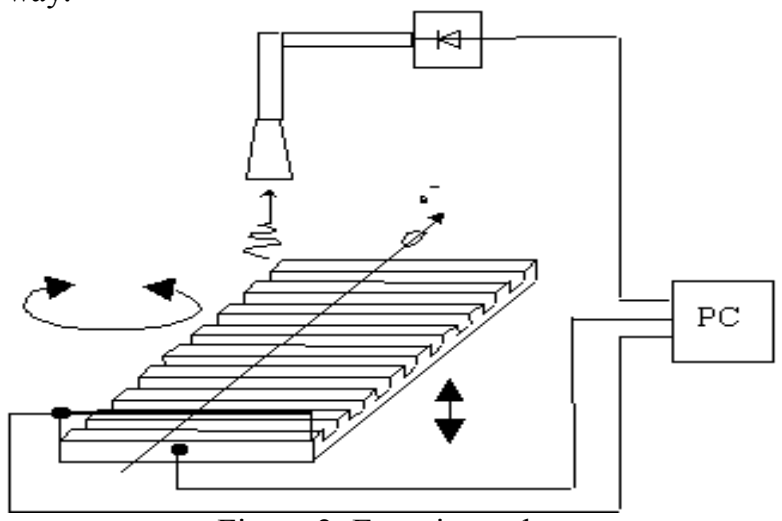

Figure 2: Experiment layout

To check ability of the proposed device to monitor the bunch length a series of simulations and experiments were done using the linear electron accelerator LIC [5] as a base research facility. The accelerator is applied for investigations in the field of high-brightness electron beam shaping and accelerating, short-wave radiation generation and interaction of a relativistic beam with plasma. The accelerator operating frequency is $2797.15 \mathrm{MHz}$. The main accelerator feature is the small beam emittance for the pulse current up to $1 \mathrm{~A}$. Beam performances at the accelerator exit are shown in the table 1.

Table 1. Beam parameters

\begin{tabular}{|l|l|}
\hline Particle energy, MeV & $13-18$ \\
\hline Pulse current duration, $\mu s$ & $0.3-1.5$ \\
\hline Pulse repetition rate, $\mathrm{Hz}$ & $1-6.25$ \\
\hline Bunch length, $\mathrm{mm}(70 \%$ of particles $)$ & $2.5-6$ \\
\hline Bunch frequency, $\mathrm{MHz}$ & 2797.15 \\
\hline Beam pulse current, $\mathrm{A}$ & $0.2-1.0$ \\
\hline Particle number per bunch & to $2 \cdot 10^{9}$ \\
\hline RMS beam size, $\mathrm{mm}$ & $1.5-2.5$ \\
\hline Beam divergence, $\mathrm{mrad}$ & $<1.0$ \\
\hline
\end{tabular}

The simulations of bunch characteristics at the linac exit were carried out with the PARMELA code [6].

\section{SIMULATION RESULTS}

We are going to use Smith-Purcell radiation observed at the fixed angle for bunch monitoring. Therefore accordance of the bunch form factor maximum at the specified wavelength to the bunch length minimum was the main question needed to be cleared. It was also necessary to determine the suitable harmonic of the linac operation frequency to be used for bunch length monitoring.

The simulations allow to determine the dependence of bunch length at the grating position on phase shift between a RF gun and the accelerating section. At the same time dependencies of the maximal instant bunch current and form factors for some harmonics on the phase shift were investigated. Results of the simulations are represented in Fig. 3.

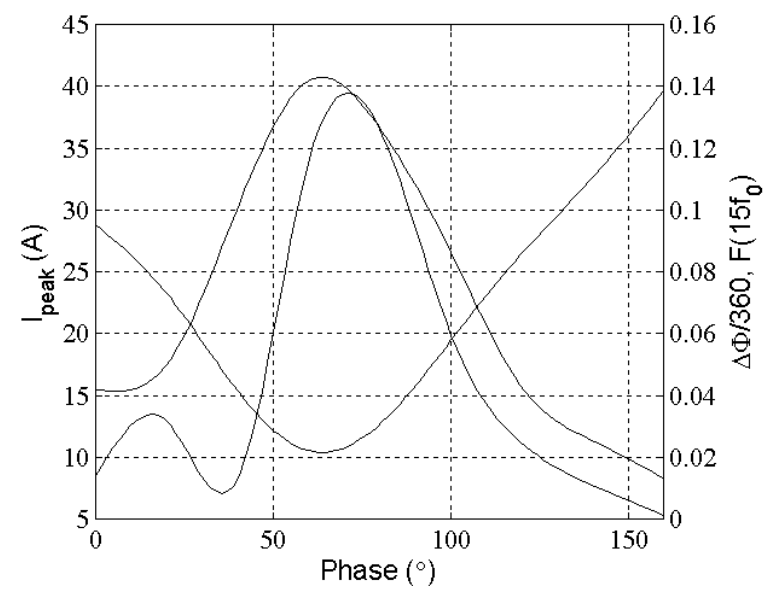

Figure 3: Dependencies of the maximal instant current (1), the bunch form factor for the $15^{\text {th }}$ harmonic of bunch repetition frequency $f_{0}(2)$ and normalised bunch phase length (3) on phase shift.

One can see in the Fig. 2 that the form factor of the $15^{\text {th }}$ harmonic $(\lambda=7.145 \mathrm{~mm})$ is enough high and its maximum is in a good agreement with the minimum of bunch phase length. The simulations also show that the maximum of a sum of bunch form factors for a large amount of harmonics (for example from 14 through 34) corresponds to the minimum of the bunch length more precisely. Nevertheless it is clear that the $15^{\text {th }}$ harmonic can be used for adjustment of the linac.

\section{EXPEREMENTAL RESULTS}

For preliminary experiments a copper grating with a period of $8 \mathrm{~mm}$, a groove depth of $4 \mathrm{~mm}$ and period number of 10 was used. The experiments were done to investigate characteristics of the Smith - Purcell radiation at the perpendicular direction to the grating.

The radiation power depending on the parameter $b$ for the pulse current of $0.75 \mathrm{~A}\left(N \cong 1.810^{9}\right)$ and electron energy of $13 \mathrm{MeV}$ was measured during the first stage of experiments. Transversal size of a beam (FWHM) at a grating position was $2.5 \mathrm{~mm}$. Fig. 4 shows the detector voltage dependence on the parameter $b$. The detected radiation power reached $10 \mathrm{~mW}$ that corresponds to $\mathrm{rf}$ 
power flow density more than $6 \mathrm{~W} / \mathrm{sr}$. This value is enhanced more than by six order of magnitude of the power flow density of the incoherent beam radiation. It is necessary to note that the narrow peak of the curve in Fig. 4 can not be explained by Eq. (2). One can see that for $\gamma=26$ dependence of radiating intensity on the $b$ is almost linear. We carried out additional experiments to study this feature.

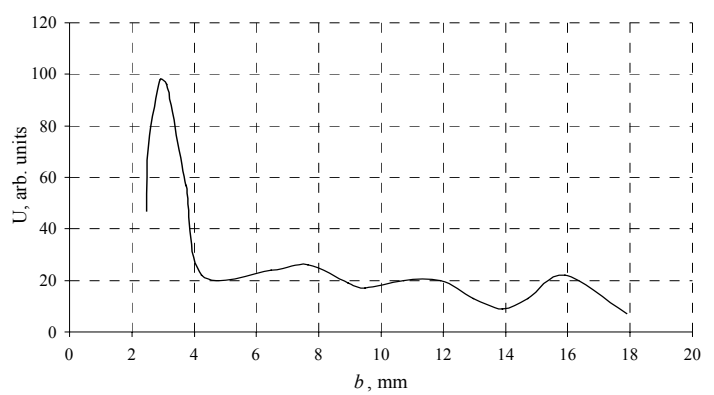

Figure 4: Detector voltage dependence on the parameter $b$.

The experiments showed existing of the millimeter radiation in the linac beam pipe regardless of the grating presence. The radiation power measured at $1 \mathrm{~m}$ far from the beam axis (Fig. 2) was about $1 \mathrm{~mW}$, in case when a metallic screen covered the grating. In our opinion the observed radiation is the coherent radiation from relativistic electron bunches in places where the beam pipe has discontinuities. To approve this we investigated radiation from a glass gap placed in the beam pipe upstream of the diffraction grating. When a wave-guide section with cut-off wavelength of $7.2 \mathrm{~mm}$ was installed between the aerial and the detector we observed strong dependence of the radiation intensity on phase shift between the injector and the accelerating structure (see Fig. 5).

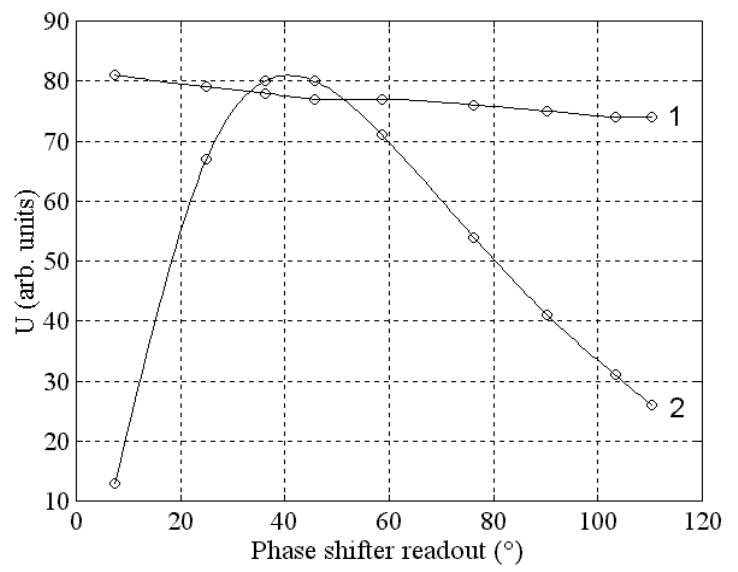

Figure 5: Dependencies of a linac output current (1) and a detector voltage (2) on phase shift.

It is obvious that behavior of a detector voltage does not correspond with behavior of an output pulse current that is evidence of bunch length changing.

Thus, the radiation observed in experiments consists of the radiation generated in the beam pipe and of the radiation from the grating. The carried out experiment confirmed that bunches at the LIC exit are enough short so a diffracting grating with a smaller period $(d=7.145 \mathrm{~mm})$ was used at the next stage. Fig. 6 shows dependence of radiation intensity at the front of grating on phase shift between the injector and the accelerating section. Output current of the linac was some high than in the previous experiment.

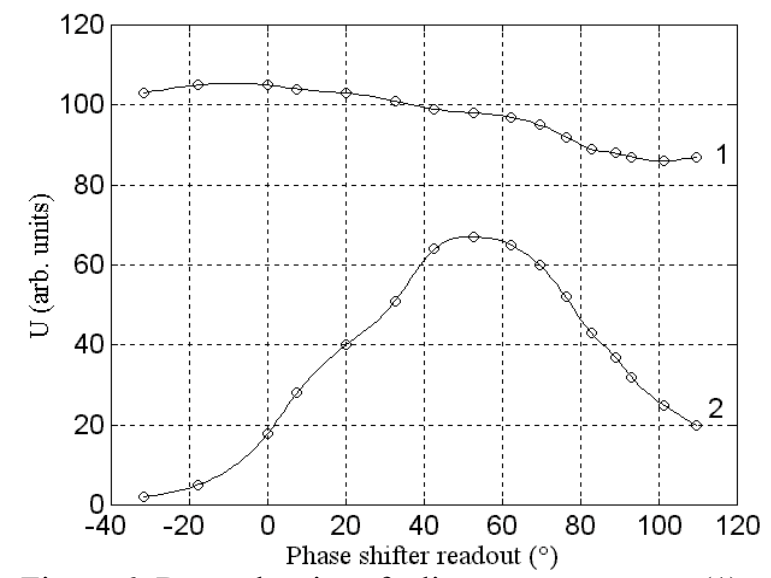

Figure 6: Dependencies of a linac output current (1) and radiation intensity at the front of grating (2) on phase shift.

It can be seen that simulation and experimental results are in good accordance (see Fig. 3, 5 and 6). Therefore an optimal tuning of the accelerator can be made using a detected signal that is proportional to radiation intensity as an indicator.

\section{CONCLUSION}

On the basis of received results following conclusions can be made. The intensity of the observed radiation is higher significantly than incoherent radiation intensity and is determined by a bunch length. The observed radiation consists of coherent radiation from the grating and of coherent radiation from discontinuities of a beam pipe. The described technique can be used as a bunch length monitor to choose the optimal conditions of a relativistic bunch shaping and accelerating.

\section{REFERENCES}

[1] S.J. Smith and E.M. Purcell, "Visible Light from Localized Surface Charges Moving across a Grating”, Phys. Rev., vol.2, No.4, pp.1069, 1953.

[2] Y. Shibata et. al., "Coherent Smith-Purcell Radiation in the Millimeter-Wave Region from a Short-Bunch Beam of Relativistic Electrons", Phys. Rev. E, Vol. 57, pp. 1061-1074, 1998.

[3] B.M. Bolotovsky, G.V. Voskresensky, "Diffraction radiation", Uspechi Phys. Nauk, 88, (2), pp. 209-229. 1966 (in Russian).

[4] D. C. Nguyen, "Electron Bunch Length Diagnostic with Coherent Smith-Purcell Radiation", Proc. of the 1997 PAC Conf. pp. $1990-$ 1992, 1997.

[5] M.I. Ayzatsky et al., "Operating Performances and Current Status of the Laser Injector Complex Facility (LIC)", Proc. of the XVIII LINAC Conf. Geneva, Switzerland, pp. 116-118, 1996.

[6] L.M. Young, "PARMELA", Los Alamos National Laboratory, LAUR-96-1835, 1996. 